\title{
Nomogram for Predicting Survival in Advanced Gastric Cancer after Neoadjuvant Chemotherapy and Radical Surgery
}

\author{
Yonghe Chen $\mathbb{D}^{1,2}$ Dan Liu $\mathbb{D}^{1,}{ }^{3}$ Jian Xiao $\mathbb{D}^{2,4}$ Jun Xiang $\mathbb{D}^{1,2}$ Aihong Liu, ${ }^{1,2}$ Shi Chen, ${ }^{1,2}$ \\ Junjie Liu $\mathbb{D}^{1,2}$ Xiansheng $\mathrm{Hu}^{1,2}$ and Junsheng Peng $\mathbb{D}^{1,2}$ \\ ${ }^{1}$ Department of Gastric Surgery, The Sixth Affiliated Hospital, Sun Yat-sen University, Guangzhou 510655, China \\ ${ }^{2}$ Guangdong Institute of Gastroenterology, Guangdong Provincial Key Laboratory of Colorectal and Pelvic Floor Diseases, \\ Guangzhou 510655, China \\ ${ }^{3}$ Department of Laboratory Science, The Second Affiliated Hospital of Guangzhou University of Chinese Medicine, \\ Guangzhou 510105, China \\ ${ }^{4}$ Department of Medical Oncology, The Sixth Affiliated Hospital, Sun Yat-sen University, Guangzhou 510655, China
}

Correspondence should be addressed to Junsheng Peng; pengjsh@mail.sysu.edu.cn

Received 26 April 2021; Revised 9 June 2021; Accepted 10 July 2021; Published 28 July 2021

Academic Editor: Fausto Rosa

Copyright (C) 2021 Yonghe Chen et al. This is an open access article distributed under the Creative Commons Attribution License, which permits unrestricted use, distribution, and reproduction in any medium, provided the original work is properly cited.

\begin{abstract}
Background. Neoadjuvant chemotherapy (NAC) with subsequent radical surgery has become a popular treatment modality for advanced gastric cancer (AGC) worldwide. However, the survival benefit is still controversial, and prognostic factors remain undetermined. Aim. To identify clinical parameters that are associated with the survival of AGC patients after NAC and radical surgery and to establish a nomogram integrating multiple factors to predict survival. Methods. We reviewed the medical profiles of 215 AGC patients who received NAC and radical resection, and clinical parameters concerning NAC, surgery, pathological findings, and adjuvant chemotherapy were analyzed using a Cox regression model to determine their impact on survival. Based on these factors, a nomogram was developed and validated. Results. The overall 1-year and 3-year survival rates were $85.8 \%$ and $55.6 \%$, respectively. Younger age ( $<60$ years old), increased examined lymph nodes (exLNs), successful R0 resection, the achievement of pathological complete response (pCR), and acceptance of adjuvant chemotherapy were positive predictors of survival. The C-index of the established nomogram was 0.785 . The area under receiver operating curve (ROC) at $1 / 3$ years of prediction was 0.694/0.736, respectively. The model showed an ideal calibration following internal bootstrap validation. Conclusion. A nomogram predicting survival after NAC and surgery was established. Since this nomogram exhibited satisfactory and stable predictive power, it can be inferred that this is a practical tool for predicting AGC patient survival after NAC and radical surgery.
\end{abstract}

\section{Introduction}

Gastric cancer is the fifth most common malignancy in the world and the third leading cause of cancer-related death [1]. The majority of affected patients are diagnosed at an advanced stage due to the insidious onset of this malignancy, especially in China [2], resulting in poor survival. In recent years, neoadjuvant chemotherapy (NAC) was introduced into the integrated treatment modality for advanced gastric cancer (AGC) and soon became popular [3]. Some scholars stated that NAC could result in tumor downstaging and a higher curative resection rate and may eventually prolong survival for AGC patients [4-9]. However, some other trials failed to prove any survival benefit from NAC [10-12]. Moreover, well-designed prospective trials are still lacking. Thus, the necessity of NAC for AGC patients is still controversial [13].

To achieve precision medication, the decision of whether to administer NAC should be determined according to the potential survival gain [14]. Thus, it is important to address the survival predictors for patients who receive NAC and surgery. A few studies set out to identify the factors that predict survival after NAC [15-19]. However, these studies only addressed the impact of singular factors without systematically 
studying all possible factors or combining them. A practical model integrating multiple factors to predict survival after NAC and surgery is still lacking.

Therefore, the purpose of this study was to identify clinical parameters that have predictive value on survival and their weights of impact. Second, we aimed to establish a nomogram integrating multiple factors to predict survival in an attempt to offer a larger picture of survival after the combined treatment modality of NAC with subsequent radical surgery and to provide a practical tool for clinical use.

\section{Materials and Methods}

2.1. Study Population and Data Collection. In the initial screening process, we identified 1346 patients who underwent radical gastrectomy from the gastric cancer database of The Sixth Affiliated Hospital, Sun Yat-sen University, from March 2012 to December 2019.

The inclusion criteria were as follows: (i) histologically confirmed adenocarcinoma of the stomach or esophagogastric junction; (ii) a clinical stage of $\mathrm{T} 3 \mathrm{~N}+$ or $\mathrm{T} 4 \mathrm{NO} /+$ as evaluated by computed tomography imaging; (iii) administration of NAC followed by gastrectomy with standardized D2 lymphadenectomy. The exclusion criterion was as follows: (i) incomplete clinical data; (ii) lost follow-up after surgery; (iii) preoperative radiation therapy. The inclusion and exclusion process is depicted in Figure 1.

Finally, a total of 215 patients were included in this study. Information on demographics, including sex, age, body mass index (BMI), biopsy pathological tumor grade, and clinical stage, was collected.

This retrospective study was approved by the Institutional Review Board of The Sixth Affiliated Hospital, Sun Yat-sen University.

2.2. Neoadjuvant Chemotherapy. All patients enrolled received neoadjuvant chemotherapy set by the multidisciplinary team (MDT) comprising surgeons, medical oncologists, and radiologists of The Sixth Affiliated Hospital, Sun Yat-sen University. Information on regimen, cycles, and timing was collected.

2.3. Surgery. All patients received subsequent curative tumor resection (total or subtotal gastrectomy) with D2 lymphadenectomy. Open or laparoscopic surgery was chosen according to the preference of the surgeon. A thorough examination of the abdominal cavity was routinely performed to determine the status of peritoneal metastasis, while a peritoneal washing cytology test was not routinely conducted. Information on gastrectomy extent, resection approach, surgical findings, postsurgery complications, and pathological findings was collected.

2.4. Adjuvant Chemotherapy. Information on completion, regimen, cycles, and timing of adjuvant chemotherapy was collected.

2.5. Follow-Up. All follow-up work was conducted by the follow-up office from the gastric cancer database. Information on survival was retrieved.

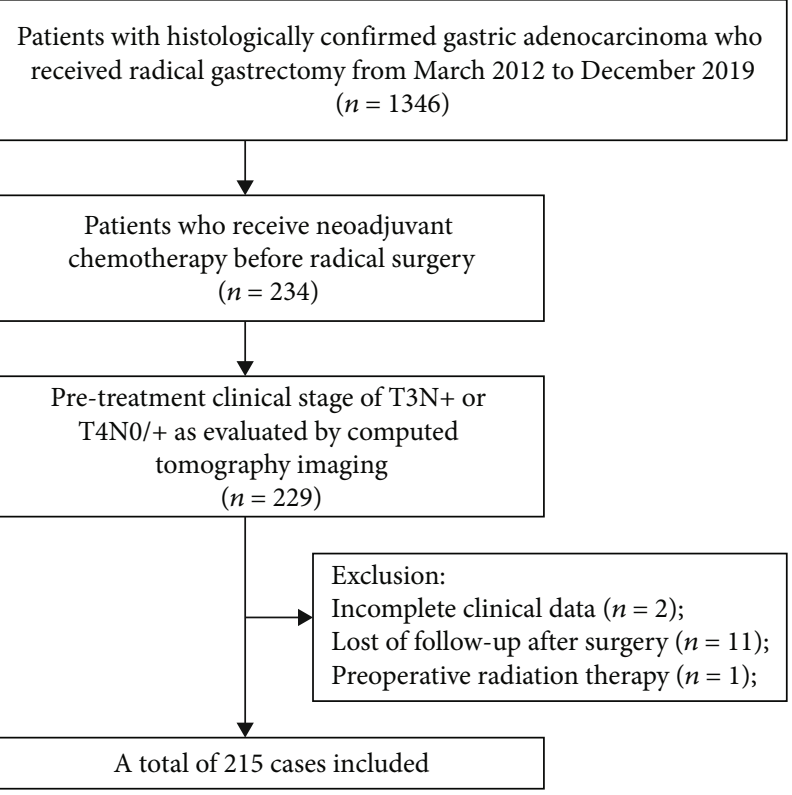

Figure 1: Flow chart of the inclusion and exclusion process.

2.6. Data Analysis. The normality of the data was assessed using the Kolmogorov-Smirnov test and normal probability plots. Parameters that were not normally distributed are expressed in the form of medians (upper quartile to lower quartile). Both categorical and continuous variables were analyzed independently with a Cox regression model, and those variables with a $p$ value $<0.05$ were then enrolled in the multivariable analysis. A stepwise selection method was adopted to develop a regression model with maximum predictive power. A nomogram was then developed upon the established model. The concordance statistic was acquired for the nomogram, and internal validation using the bootstrap method was performed to determine the adjusted concordance statistic. A calibration curve and receiver operating curve (ROC) for the nomogram were generated to show the prediction efficiency of the model. All statistical analyses were performed using SPSS software ver. 22.0 (IBM, Armonk, NY, USA) and R version 3.6.1 software (The R Foundation for Statistical Computing, Vienna, Austria; http://www.r-project.org/).

\section{Results}

A total of 215 patients with a histological diagnosis of adenocarcinoma of the stomach or esophagogastric junction from March 2012 to December 2019 were enrolled in the study. As depicted in Table 1, the majority of patients were male $(167 / 215,77.7 \%)$. The average age of the cohort was $57 \pm 11$ years old. Tumors were predominantly poorly differentiated (grade 3, 148/215, 68.8\%), with radiologically suspicious lymph node metastasis $(210 / 215,97.7 \%)$.

All patients received treatments as depicted in Table 2, including NAC, surgery, and adjuvant chemotherapy. Patients received a median of 4 cycles of NAC. More than half $(121 / 215,56.3 \%)$ of the patients received the mFLOT regimen, and other mainstream regimens included SOX (60/215, 
TABle 1: Patient characteristics, hazard ratios, and $p$ values from univariable survival analysis $(n=215)$.

\begin{tabular}{|c|c|c|c|}
\hline & $n(\%)$ & Hazard ratio $(95 \% \mathrm{CI})$ & $p$ value \\
\hline \multicolumn{4}{|l|}{ Age (years) } \\
\hline$<60$ & $113(52.6 \%)$ & Reference & - \\
\hline$\geq 60$ & $102(47.4 \%)$ & $2.34(1.23-4.46)$ & 0.01 \\
\hline \multicolumn{4}{|l|}{ Sex } \\
\hline Male & $167(77.7 \%)$ & Reference & - \\
\hline Female & $48(22.3 \%)$ & $1.49(0.75-2.94)$ & 0.26 \\
\hline BMI $\left(\mathrm{kg} / \mathrm{m}^{2}\right)$ & $21.8 \pm 2.9$ & $0.99(0.89-1.11)$ & 0.87 \\
\hline $0-24$ & $173(80.5 \%)$ & Reference & - \\
\hline$>24$ & $42(19.5 \%)$ & $0.73(0.31-1.75)$ & 0.48 \\
\hline \multicolumn{4}{|l|}{ Location } \\
\hline Upper & $85(39.5 \%)$ & Reference & - \\
\hline Middle & $38(17.7 \%)$ & $0.96(0.40-2.31)$ & 0.93 \\
\hline Lower & $85(39.5 \%)$ & $0.73(0.36-1.47)$ & 0.38 \\
\hline Whole & $7(3.3 \%)$ & $0.005(0-10+)$ & 0.72 \\
\hline \multicolumn{4}{|l|}{ Tumor grade } \\
\hline G3 & $151(70.2 \%)$ & Reference & - \\
\hline G2 & $56(26 \%)$ & $0.78(0.38-1.62)$ & 0.51 \\
\hline G1 & $8(3.7 \%)$ & $0.006(0-10+)$ & 0.65 \\
\hline \multicolumn{4}{|c|}{ Clinical T stage } \\
\hline$c T 3$ & $109(50.7 \%)$ & Reference & - \\
\hline$c T 4$ & $106(49.3 \%)$ & $1.61(0.83-3.16)$ & 0.16 \\
\hline \multicolumn{4}{|c|}{ Clinical N staging } \\
\hline cNO & $5(2.3 \%)$ & Reference & - \\
\hline$c N+$ & $210(97.7 \%)$ & $1.61(0.21-12.21)$ & 0.645 \\
\hline
\end{tabular}

27.9\%), FOLFOX (11/215, 5.1\%), and XELOX (7/215, 3.3\%). Subsequent radical resection followed after an average of 29 \pm 10 days. The most common resection approach was laparoscopy. A thorough abdominal exploration was routinely conducted for all patients before resection, and 3.3\% (7/215) of patients were confirmed to have occult distal metastasis that was not identified before surgery. Additionally, $11.6 \%$ $(25 / 215)$ of patients received multivisceral resection due to adjacent organ invasion or distal metastasis, but in the end, R0 resection was achieved for most patients (190/215, $88.4 \%$ ). Major complications (Grade IIIa-V according to the Clavien-Dindo Classification system) included anastomotic leakage $(11 / 215,5.1 \%)$, bleeding $(7 / 215,3.3 \%)$, thoracic effusion $(4 / 215,1.9 \%)$, ileus $(2 / 215,0.9 \%)$, and severe pneumonia $(1 / 215,0.5 \%)$. Nineteen patients were managed by medication therapy or interventions that require no general anesthesia, such as endoscopic hemostasis or ultrasound-guided centesis drainage, and 5 patients were managed by reoperation. One patient eventually died of severe pneumonia after being transmitted to the intensive care unit. After radical resection, the majority of patients received a median of 5 cycles of adjuvant chemotherapy (198/215, 92.1\%), mostly FOLFOX (79/215, $36.7 \%)$ or its derived regimen SOX $(51 / 215,23.7 \%)$; others included a docetaxel-based regimen $(42 / 215,19.5 \%)$ and oral fluorouracil $(26 / 215,12.1 \%)$.
The pathological findings are depicted in Table 3. Pathological complete response (pCR) was achieved in 13\% $(28 / 215)$ of patients. The average number of examined lymph nodes (exLNs) was $26 \pm 13$, and half of the patients (117/215, $54.4 \%$ ) had lymph node metastasis in the final pathological analysis, significantly less than estimated presurgically $(\mathrm{cN}$ t: 210/215, 97.7\%).

In the survival analysis, the median follow-up duration of the cohort was 12 (5-21) months, with 39 cases of tumorrelated death observed during this period. The overall 1-year and 3-year survival rates were $85.8 \%$ and $55.6 \%$, respectively. Tables 1-3 show the hazard ratios, $95 \%$ confidence intervals, and the respective $p$ value of each parameter in univariable survival analysis. Parameters that had a significant impact on survival $(p<0.05)$ were age, R0 resection, vascular tumor embolus, nerve invasion, pCR, number of exLNs, and adjuvant chemotherapy. These parameters were enrolled in multivariable analysis and selected by the stepwise procedure to build a model with the strongest predictive power (Akaike information criterion statistic $=320$ ). In the final established Cox proportional hazards model, younger age ( $<60$ years old), increased exLNs, successful R0 resection, $\mathrm{pCR}$, and receiving adjuvant chemotherapy were predictors for prolonged survival. Table 4 summarizes the hazard ratio and $95 \%$ confidence interval of each predictor. A nomogram predicting survival after NAC and radical resection was constructed according to the established model, as shown in Figure 2. The apparent C-index of the nomogram was 0.785 , indicating a satisfactory efficiency in predicting survival. Calibration curves demonstrated a good fitting between predicted and actual observations of survival, indicating an ideal statistical performance for predicting survival, as shown in Figure 3. The areas under the curve (AUC) of receiver operating curve (ROC) at $1 / 3$ years were $0.694 / 0.736$, respectively, showing a good discriminative power of the model (Figure 4).

\section{Discussion}

In our study, we found that younger age ( $<60$ years old), increased exLNs, successful R0 resection, $\mathrm{pCR}$, and receiving adjuvant chemotherapy were positive predictors of survival. A nomogram was established according to the weights of these predictors in the model, with exLNs and pCR having the largest impact on survival. All factors included in the model are easily available in clinical practice, and internal validation showed consistent and stable predictive power, making it a practical tool for clinical reference.

There have been many studies trying to identify survival predictors for AGC patients who received NAC and radical surgery, but most of these studies only show which factors may be related to survival without specifying the weights and impact of these factors; a panoramic model that describes the survival of this patient subgroup is lacking. Nomogram is a prediction model based on a Cox regression model consisted of axes and a scoring system, each axis represents an independent survival predictor, and the corresponding score on the axis represents the impact of the predictor. It gives an easily perceptible visualization of the survival of a specific disease. To our knowledge, this is the 
TABLE 2: Treatment information on chemotherapy and surgery, hazard ratios, and $p$ values from univariable survival analysis $(n=215)$.

\begin{tabular}{|c|c|c|c|}
\hline & $n(\%)$ & Hazard ratio $(95 \% \mathrm{CI})$ & $p$ value \\
\hline \multicolumn{4}{|l|}{ Neoadjuvant regimen } \\
\hline$m F L O T$ & $121(56.3 \%)$ & Reference & \\
\hline SOX, XELOX or FOLOFX & $78(36.3 \%)$ & $0.45(0.19-1.05)$ & 0.07 \\
\hline Other & $16(7.4 \%)$ & $0.39(0.15-1.02)$ & 0.05 \\
\hline Cycles received & $4(4-4)$ & $1.08(0.88-1.34)$ & 0.45 \\
\hline Time gap between NAC and surgery (days) & $29 \pm 10$ & $1.03(1-1.05)$ & 0.07 \\
\hline \multicolumn{4}{|l|}{ Resection extend } \\
\hline Total gastrectomy & $129(60 \%)$ & Reference & - \\
\hline Subtotal gastrectomy & $86(40 \%)$ & $0.76(0.39-1.47)$ & 0.42 \\
\hline \multicolumn{4}{|l|}{ Laparoscopic surgery } \\
\hline Yes & $167(77.7 \%)$ & Reference & - \\
\hline No & $48(22.3 \%)$ & $1.45(0.76-2.78)$ & 0.26 \\
\hline \multicolumn{4}{|l|}{ Metastasis found during surgery } \\
\hline Yes & $7(3.3 \%)$ & Reference & - \\
\hline No & $208(96.7 \%)$ & $0.83(0.11-6.08)$ & 0.83 \\
\hline \multicolumn{4}{|l|}{ Multivisceral resection } \\
\hline Yes & $25(11.6 \%)$ & Reference & - \\
\hline No & $190(88.4 \%)$ & $0.64(0.26-1.54)$ & 0.32 \\
\hline \multicolumn{4}{|l|}{ R0 resection } \\
\hline Yes & $190(88.4 \%)$ & Reference & - \\
\hline No & $25(11.6 \%)$ & $0.40(0.20-0.83)$ & 0.01 \\
\hline \multicolumn{4}{|l|}{ Major complication* } \\
\hline No & $191(88.8 \%)$ & Reference & - \\
\hline IIIa & $24(11.2 \%)$ & $0.67(0.21-2.18)$ & 0.51 \\
\hline \multicolumn{4}{|l|}{ Reoperation within 30 days } \\
\hline Yes & $5(2.3 \%)$ & Reference & - \\
\hline No & $210(97.7 \%)$ & $0.72(0.19-10+)$ & 0.75 \\
\hline \multicolumn{4}{|l|}{ Adjuvant chemotherapy } \\
\hline No & $17(7.9 \%)$ & Reference & - \\
\hline Yes & $198(92.1 \%)$ & $0.27(0.12-0.62)$ & $<0.01$ \\
\hline Cycles received & $5(3-5)$ & $0.88(0.78-1)$ & 0.05 \\
\hline Time between surgery and adjuvant chemotherapy (days) & $36 \pm 19$ & $1.03(1-1.05)$ & 0.07 \\
\hline
\end{tabular}

mFLOT: docetaxel $50 \sim 60 \mathrm{mg} / \mathrm{m}^{2}+$ oxaliplatin $85 \mathrm{mg} / \mathrm{m}^{2}+$ fluorouracil $2800 \mathrm{mg} / \mathrm{m}^{2}$ iv over 48 hours, every 2 weeks; SOX: oxaliplatin $130 \mathrm{mg} / \mathrm{m}^{2}$ iv + tegafur/gimeracil/oteracil potassium capsule $40 \sim 60 \mathrm{mg}$ bid D1-D14 every 3 weeks; XELOX: oxaliplatin $130 \mathrm{mg} / \mathrm{m}^{2}+$ capecitabine $1000 \mathrm{mg} / \mathrm{m}^{2}$ bid D1D14 every 3 weeks; FOLFOX: oxaliplatin $85 \mathrm{mg} / \mathrm{m}^{2}+$ fluorouracil $2800 \mathrm{mg} / \mathrm{m}^{2}$ civ over 48 hours every 2 weeks. The dosage of the regimens above might be modified according to the preference of the oncologist. NAC: neoadjuvant chemotherapy. ${ }^{*}$ Major complication is defined according to the Clavien-Dindo Classification system (Grade III and above): Grade III, complications requiring surgical, endoscopic, or radiological intervention (IIIa: no general anesthesia required; IIIb: general anesthesia required); Grade IV, life-threatening complications requiring IC/ICU management; Grade V, death.

first nomogram integrating clinical parameters from different treatment phases for AGC patients after NAC and surgery. Most of the parameters enrolled in the final model emphasize the importance of complete elimination of the tumor. First, for the number of exLNs, according to the $8^{\text {th }}$ edition of the AJCC staging manual, the retrieval of at least 16 lymph nodes is the minimal requirement for lymph node dissection [20], but there is growing evidence that increasing the number of harvested lymph nodes significantly improves survival [21-28]. Increased exLNs indicate a more extended lymph node dissection and a more thorough clearance of the tumor. As shown in our model, exLNs are positively rel- ative to the survival, with the longest axis, indicating the great importance of complete tumor clearance. Second, pCR is another significant positive predictor of survival in our model, with the second-longest axis (non-pCR vs. pCR, HR: 9.06, 95\% CI: 1.22-67.4). It has been well established that pCR is closely related to prolonged survival after NAC and radical resection [29-31]. Previous data showed that those with pCR following NAC could achieve a 5-year survival rate of up to $89 \%$, which is very favorable among AGC patients [20]. Thus, NAC is highly recommended for patients with the potential of achieving pCR. However, pCR is uncommon, and our data showed that only $13 \%$ of patients achieve pCR, 
TABLE 3: Pathological findings, hazard ratios, and $p$ values from univariable survival analysis $(n=215)$.

\begin{tabular}{|c|c|c|c|}
\hline & $n(\%)$ & Hazard ratio $(95 \% \mathrm{CI})$ & $p$ value \\
\hline \multicolumn{4}{|c|}{ Pathological complete response } \\
\hline No & $187(87 \%)$ & Reference & - \\
\hline Yes & $28(13 \%)$ & $0.1(0.01-0.77)$ & 0.03 \\
\hline \multicolumn{4}{|l|}{ ypT stage } \\
\hline TO & $28(13 \%)$ & $0.09(0.01-0.66)$ & 0.02 \\
\hline$T 1$ & $26(12.1 \%)$ & $0.46(0.14-1.52)$ & 0.20 \\
\hline$T 2$ & $21(9.8 \%)$ & $0.43(0.13-1.44)$ & 0.17 \\
\hline T3 & $136(63.3 \%)$ & Reference & - \\
\hline T4 & $4(1.9 \%)$ & $1.33(0.3-5.92)$ & 0.70 \\
\hline Harvested lymph nodes & $26 \pm 13$ & $0.95(0.92-0.98)$ & $<0.01$ \\
\hline \multicolumn{4}{|l|}{ ypN stage } \\
\hline NO & $98(45.6 \%)$ & Reference & - \\
\hline N1 & $51(23.7 \%)$ & $1.83(0.81-4.14)$ & 0.15 \\
\hline N2 & $33(15.3 \%)$ & $2.0(0.74-5.43)$ & 0.17 \\
\hline N3a & $26(12.1 \%)$ & $3.64(1.42-9.35)$ & $<0.01$ \\
\hline$N 3 b$ & $7(3.3 \%)$ & $4.89(1.36-17.6)$ & 0.02 \\
\hline \multicolumn{4}{|l|}{ Vascular tumor embolus } \\
\hline No & $175(81.4 \%)$ & Reference & - \\
\hline Yes & $40(18.6 \%)$ & $2.31(1.12-4.78)$ & 0.02 \\
\hline \multicolumn{4}{|l|}{ Nerve invasion } \\
\hline No & $143(66.5 \%)$ & Reference & - \\
\hline Yes & $72(33.5 \%)$ & $2.03(1.07-3.84)$ & 0.03 \\
\hline \multicolumn{4}{|l|}{ HER2 } \\
\hline$(-)$ & 117 (54.4\%) & Reference & - \\
\hline$(+) \sim(++)$ & $53(24.7 \%)$ & $1.22(0.6-2.46)$ & 0.58 \\
\hline$(+++)$ & $4(1.9 \%)$ & $5.57(1.63-19)$ & $<0.01$ \\
\hline Data missing & $41(19.1 \%)$ & & \\
\hline
\end{tabular}

similar to previous reports (8.4-17.4\%) [32-35]. A few studies have been devoted to identifying the predictive factors of pCR; possible positive factors include good differentiation of the tumor cells, higher carcinoma embryonic antigen levels and lymphocyte ratios, and lower monocyte counts [36, 37]. Patients with these clinical features tend to benefit more from NAC. Third, evidence in recent years has shown that R0 resection is potentially beneficial for AGC patients, even those with limited metastasis or at the cost of multivisceral resection $[38,39]$, and the target of NAC is also to achieve R0 resection with minimal morbidity and mortality and eventually prolonged survival and better quality of life [40]. In our study, limited occult metastasis was discovered in $3.3 \%(7 / 215)$ of patients, and multivisceral resection was performed in $11.6 \%(25 / 215)$ of patients due to adjacent organ invasion or limited distal metastasis, but their survival did not seem to significantly deteriorate as long as R0 resection could be achieved, reemphasizing the importance of complete resection of the tumor. Last, the importance of adjuvant chemotherapy for AGC after radical resection has been well recognized for years [41]. Adjuvant chemotherapy helps to eliminate residual tumor cells and increase the chance for disease-free survival. As shown in our model, adjuvant
TABLE 4: Hazard ratio, 95\% confidence interval, and $p$ value of each predictor in the Cox regression model $(n=215)$.

\begin{tabular}{lccc}
\hline & Hazard ratio & $95 \%$ CI & $p$-value \\
\hline Age & & & \\
$\quad \geq 60$ years & 1.76 & $0.9-3.43$ & 0.09 \\
$\quad<60$ years & & Reference & \\
PCR (No vs Yes) & & & \\
$\quad$ No & 9.06 & $1.22-67.4$ & 0.03 \\
$\quad$ Yes & & Reference & \\
R0 resection (No vs Yes) & & & \\
$\quad$ No & 2.31 & $1.11-4.83$ & 0.03 \\
$\quad$ Yes & & Reference & \\
Adjuvant chemotherapy & & & \\
(Yes vs No) & & & \\
$\quad$ Yes & 0.33 & $0.14-0.79$ & 0.01 \\
$\quad$ No & & Reference & \\
exLNs (Increase per 1 node) & 0.94 & $0.91-0.98$ & $<0.01$ \\
\hline
\end{tabular}




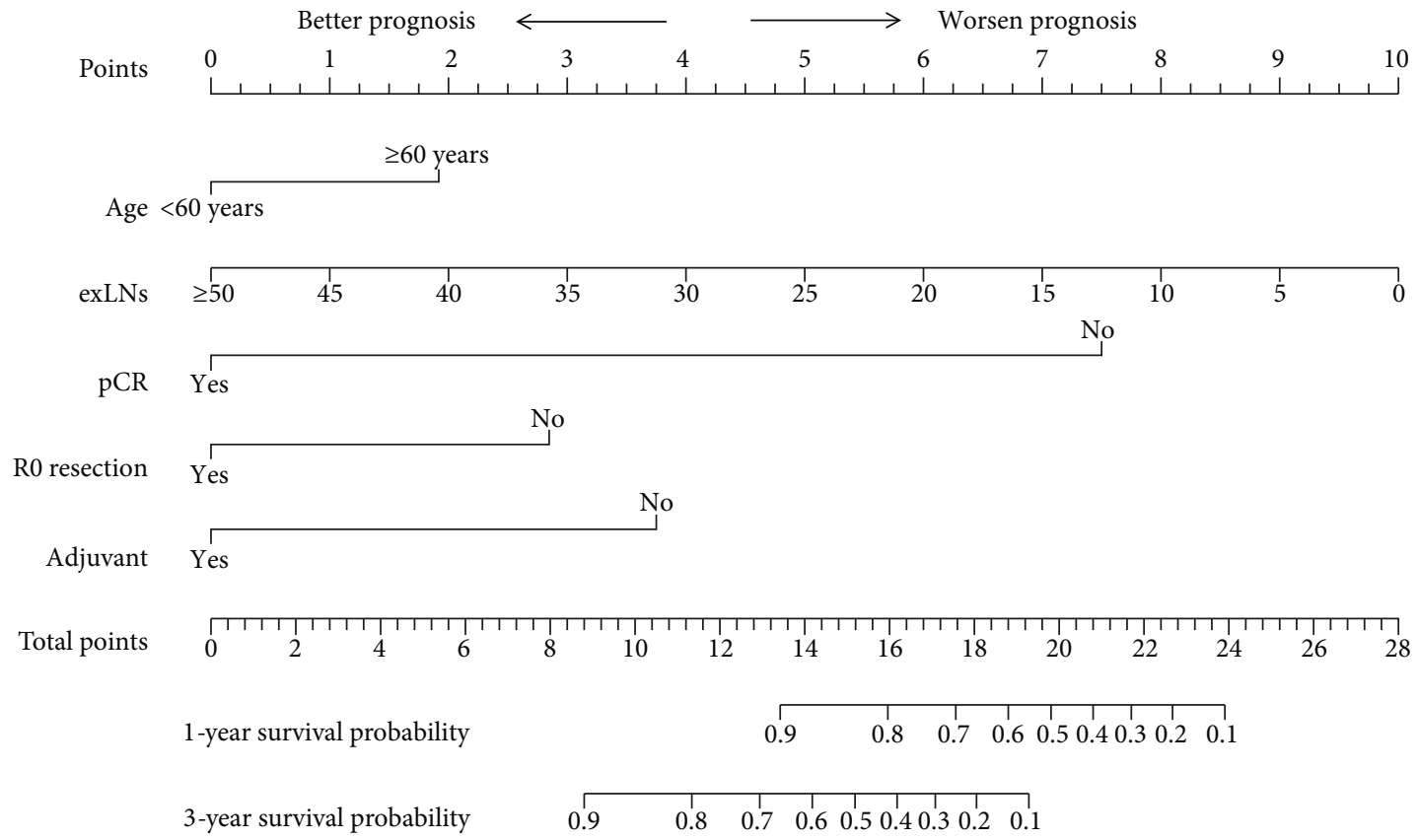

Figure 2: Parameters in Tables 1-3 with a $p$ value $<0.05$ were included in the Cox regression model to build a nomogram predicting 1- and 3year survival after neoadjuvant chemotherapy and surgery. exLNs: examined lymph nodes; pCR: pathological complete response; Adjuvant: adjuvant chemotherapy.

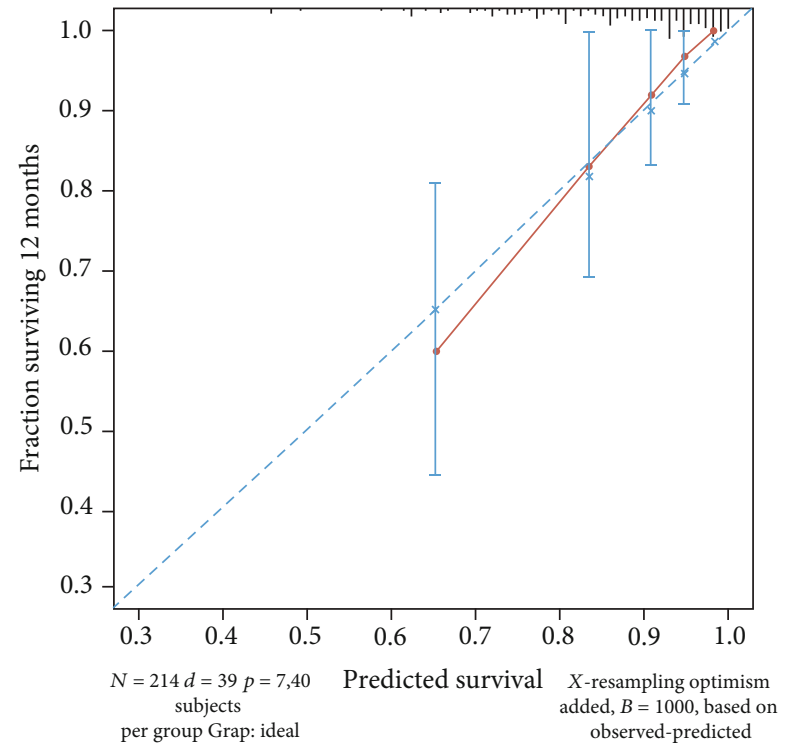

Figure 3: Calibration curve showing a good fitting of the predicted and observed survival.

chemotherapy still had a positive impact on survival after NAC and radical surgery; therefore, it should be recommended as an essential part of the treatment modality.

There are a few limitations to our study. First, it is noticeable that the ypT stage and ypN stage are not included in the model; this is because the distribution of patients across different stages was very imbalanced, as shown in Table 3. This weakens the discrimination power of the survival data. Additionally, pCR is closely related to ypT and ypN stages, and

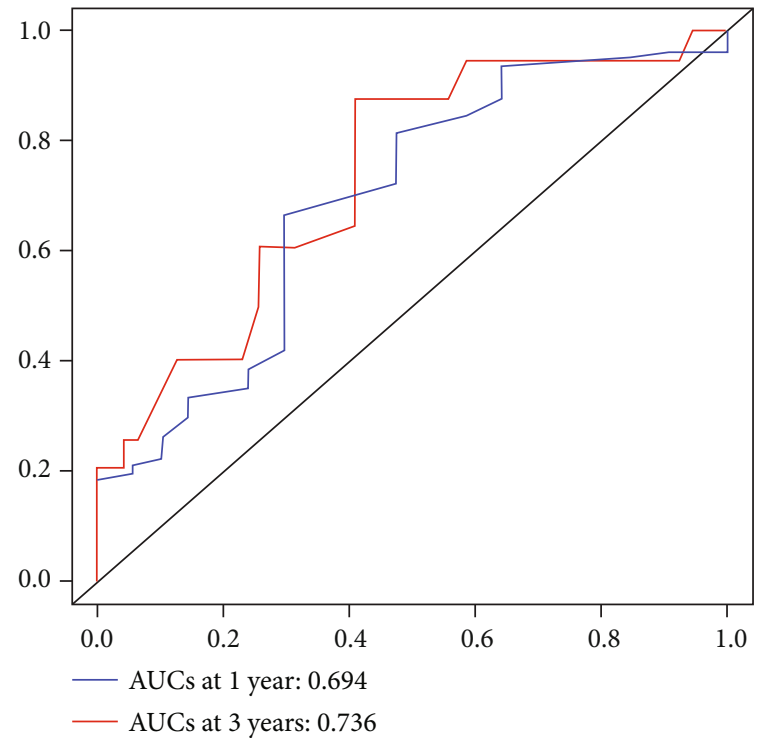

FIGURE 4: Receiver operating curve (ROC) of the nomogram at $1 / 3$ years.

including these codependent factors in the same model would have resulted in an interaction effect that would have weakened the predictive power of the model. Second, the model was validated internally using the bootstrap method, lacking external validation in an independent cohort from a different institution, but the same validation approach has been used by many previous studies and has been proven to be efficient $[42,43]$. Third, the robustness of the nomogram is limited by the sample size. 


\section{Conclusion}

A nomogram predicting the survival of AGC patients after NAC and surgery was established. To improve survival, physicians should aim at achieving pCR and R0 resection and harvesting more lymph nodes. Adjuvant chemotherapy is also highly recommended after radical resection.

\section{Data Availability}

The datasets used and analyzed during the current study are unavailable due to the privacy policy of our institution.

\section{Ethical Approval}

The study was reviewed and approved by the ethics committee of The Sixth Affiliated Hospital, Sun Yat-sen University.

\section{Consent}

All study participants, or their legal guardians, were provided with informed consent prior to study by the follow-up office.

\section{Disclosure}

A previous version of this manuscript was submitted to Research Square as a preprint (10.21203/rs.3.rs-46932/v1).

\section{Conflicts of Interest}

The authors declare that they have no competing interests.

\section{Authors' Contributions}

Peng JS and Chen YH designed the study; Chen YH, Liu D, and Xiao J contributed equally in acquiring, analyzing, and interpreting the data and drafted the initial manuscript. Xiang J, Liu AH, Chen S, Liu JJ, and Hu XS helped revise the manuscript.

\section{Acknowledgments}

This study is supported by the Research Fund of The Sixth Affiliated Hospital of Sun Yat-sen University (grant number P20200217202309876) and Nation Key Clinical Discipline.

\section{References}

[1] F. Bray, J. Ferlay, I. Soerjomataram, R. L. Siegel, L. A. Torre, and A. Jemal, "Global cancer statistics 2018: GLOBOCAN estimates of incidence and mortality worldwide for 36 cancers in 185 countries," CA: a cancer journal for clinicians, vol. 68, no. 6, pp. 394-424, 2018.

[2] L. Zong, M. Abe, Y. Seto, and J. Ji, "The challenge of screening for early gastric cancer in China," Lancet, vol. 388, no. 10060, p. 2606, 2016.

[3] N. Liu, Y. Xu, A. A. Rahnemai-Azar, D. E. Abbott, S. M. Weber, and A. O. Lidor, "National underutilization of neoadjuvant chemotherapy for gastric cancer," Journal of gastrointestinal surgery, vol. 24, no. 4, pp. 949-958, 2020.
[4] S. E. Al-Batran, R. D. Hofheinz, C. Pauligk et al., "Histopathological regression after neoadjuvant docetaxel, oxaliplatin, fluorouracil, and leucovorin versus epirubicin, cisplatin, and fluorouracil or capecitabine in patients with resectable gastric or gastro-oesophageal junction adenocarcinoma (FLOT4AIO): results from the phase 2 part of a multicentre, openlabel, randomised phase 2/3 trial," The Lancet Oncology, vol. 17, no. 12, pp. 1697-1708, 2016.

[5] S. E. Al-Batran, N. Homann, C. Pauligk et al., "Effect of neoadjuvant chemotherapy followed by surgical resection on survival in patients with limited metastatic gastric or gastroesophageal junction cancer: the AIO-FLOT3 trial," JAMA oncology, vol. 3, no. 9, pp. 1237-1244, 2017.

[6] S. E. Al-Batran, N. Homann, C. Pauligk et al., "Perioperative chemotherapy with fluorouracil plus leucovorin, oxaliplatin, and docetaxel versus fluorouracil or capecitabine plus cisplatin and epirubicin for locally advanced, resectable gastric or gastro-oesophageal junction adenocarcinoma (FLOT4): a randomised, phase 2/3 trial," Lancet, vol. 393, no. 10184, pp. 1948-1957, 2019.

[7] X. L. Wang, G. X. Wu, M. D. Zhang, M. Guo, H. Zhang, and X. F. Sun, "A favorable impact of preoperative FPLC chemotherapy on patients with gastric cardia cancer," Oncology reports, vol. 7, no. 2, pp. 241-244, 2000.

[8] M. Kano, K. Hayano, H. Hayashi et al., "Survival benefit of neoadjuvant chemotherapy with S-1 plus docetaxel for locally advanced gastric cancer: a propensity score-matched analysis," Annals of surgical oncology, vol. 26, no. 6, pp. 1805-1813, 2019.

[9] J. J. Qu, Y. R. Shi, F. R. Liu, S. Q. Ma, and F. Y. Ma, “A clinical study of paclitaxel combined with FOLFOX4 regimen as neoadjuvant chemotherapy for advanced gastric cancer," Zhonghua wei chang wai ke za zhi= Chinese journal of gastrointestinal surgery, vol. 13, no. 9, pp. 664-667, 2010.

[10] T. Kobayashi and T. Kimura, "Long-term outcome of preoperative chemotherapy with $5^{\prime}$-deoxy-5-fluorouridine $\left(5^{\prime}\right.$ DFUR) for gastric cancer," Gan to kagaku ryoho Cancer \& chemotherapy, vol. 27, no. 10, pp. 1521-1526, 2000.

[11] C. Schuhmacher, S. Gretschel, F. Lordick et al., "Neoadjuvant chemotherapy compared with surgery alone for locally advanced cancer of the stomach and cardia: European Organisation for Research and Treatment of Cancer randomized trial 40954," Journal of clinical oncology: official journal of the American Society of Clinical Oncology, vol. 28, no. 35, pp. 5210-5218, 2010.

[12] M. Imano, T. Itoh, T. Satou et al., "Prospective randomized trial of short-term neoadjuvant chemotherapy for advanced gastric cancer," European journal of surgical oncology, vol. 36, no. 10, pp. 963-968, 2010.

[13] R. Reddavid, S. Sofia, P. Chiaro et al., "Neoadjuvant chemotherapy for gastric cancer. Is it a must or a fake? World journal of gastroenterology," vol. 24, no. 2, pp. 274-289, 2018.

[14] M. Scartozzi, M. Pistelli, A. Bittoni et al., "Novel perspectives for the treatment of gastric cancer: from a global approach to a personalized strategy," Current oncology reports, vol. 12, no. 3, pp. 175-185, 2010.

[15] L. Chen, Y. Yan, L. Zhu et al., "Systemic immuneinflammation index as a useful prognostic indicator predicts survival in patients with advanced gastric cancer treated with neoadjuvant chemotherapy," Cancer management and research, vol. 9, pp. 849-867, 2017.

[16] K. Fujitani, K. Nakamura, J. Mizusawa et al., "Posttherapy topographical nodal status, ypN-site, predicts survival of 
patients who received neoadjuvant chemotherapy followed by curative surgical resection for non-type 4 locally advanced gastric cancer: supplementary analysis of JCOG1004-A," Gastric cancer, vol. 24, no. 1, pp. 197-204, 2021.

[17] N. Ikoma, J. S. Estrella, M. B. Murphy et al., "Tumor regression grade in gastric cancer after preoperative therapy," Journal of gastrointestinal surgery, vol. 25, no. 6, pp. 1380-1387, 2021.

[18] M. A. Pereira, M. F. Ramos, A. R. Dias et al., "Lymph node regression after neoadjuvant chemotherapy: a predictor of survival in gastric cancer," Journal of surgical oncology, vol. 121, no. 5, pp. 795-803, 2020.

[19] S. Lorenzen, S. Blank, F. Lordick, J. R. Siewert, and K. Ott, "Prediction of response and prognosis by a score including only pretherapeutic parameters in 410 neoadjuvant treated gastric cancer patients," Annals of surgical oncology, vol. 19, no. 7, pp. 2119-2127, 2012.

[20] M. B. Amin, F. L. Greene, S. B. Edge et al., "The eighth edition AJCC Cancer Staging Manual: continuing to build a bridge from a population-based to a more "personalized" approach to cancer staging," CA: a cancer journal for clinicians, vol. 67, no. 2, pp. 93-99, 2017.

[21] Y. Y. Liu, W. L. Fang, F. Wang et al., "Does a higher cutoff value of lymph node retrieval substantially improve survival in patients with advanced gastric cancer?-time to embrace a new digit," Oncologist, vol. 22, no. 1, pp. 97-106, 2017.

[22] S. S. Macalindong, K. H. Kim, B. H. Nam et al., "Effect of total number of harvested lymph nodes on survival outcomes after curative resection for gastric adenocarcinoma: findings from an eastern high-volume gastric cancer center," BMC Cancer, vol. 18, no. 1, p. 73, 2018.

[23] D. D. Smith, R. R. Schwarz, and R. E. Schwarz, "Impact of total lymph node count on staging and survival after gastrectomy for gastric cancer: data from a large US-population database," Journal of clinical oncology, vol. 23, no. 28, pp. 7114-7124, 2005.

[24] A. M. Bouvier, O. Haas, F. Piard, P. Roignot, C. BonithonKopp, and J. Faivre, "How many nodes must be examined to accurately stage gastric carcinomas? Results from a population based study," Cancer, vol. 94, no. 11, pp. 2862-2866, 2002.

[25] J. E. Samples, K. B. Stitzenberg, and M. O. Meyers, Lymph node yield and survival in gastric carcinoma, American Society of Clinical Oncology, 2014.

[26] C. M. Huang, J. X. Lin, C. H. Zheng, P. Li, J. W. Xie, and B. J. Lin, "Effect of negative lymph node count on survival for gastric cancer after curative distal gastrectomy," European journal of surgical oncology, vol. 37, no. 6, pp. 481-487, 2011.

[27] S. H. Kong, H. J. Lee, H. S. Ahn et al., "Stage migration effect on survival in gastric cancer surgery with extended lymphadenectomy: the reappraisal of positive lymph node ratio as a proper N-staging," Annals of surgery, vol. 255, no. 1, pp. 50-58, 2012.

[28] M. S. Karpeh, L. Leon, D. Klimstra, and M. F. Brennan, "Lymph node staging in gastric cancer: is location more important than Number? An analysis of 1, 038 patients," Annals of surgery, vol. 232, no. 3, pp. 362-371, 2000.

[29] K. Becker, J. D. Mueller, C. Schulmacher et al., "Histomorphology and grading of regression in gastric carcinoma treated with neoadjuvant chemotherapy," Cancer, vol. 98, no. 7, pp. 15211530, 2003.

[30] C. Rödel, P. Martus, T. Papadoupolos et al., "Prognostic significance of tumor regression after preoperative chemoradiotherapy for rectal cancer," Journal of clinical oncology, vol. 23, no. 34, pp. 8688-8696, 2005.
[31] M. Maas, P. J. Nelemans, V. Valentini et al., "Long-term outcome in patients with a pathological complete response after chemoradiation for rectal cancer: a pooled analysis of individual patient data," The Lancet Oncology, vol. 11, no. 9, pp. 835844, 2010.

[32] R. C. Fields, V. E. Strong, M. Gönen et al., "Recurrence and survival after pathologic complete response to preoperative therapy followed by surgery for gastric or gastrooesophageal adenocarcinoma," British journal of cancer, vol. 104, no. 12, pp. 1840-1847, 2011.

[33] S. Lorenzen, M. Hentrich, C. Haberl et al., "Split-dose docetaxel, cisplatin and leucovorin/fluorouracil as first-line therapy in advanced gastric cancer and adenocarcinoma of the gastroesophageal junction: results of a phase II trial," Annals of Oncology, vol. 18, no. 10, pp. 1673-1679, 2007.

[34] M. Stahl, M. K. Walz, M. Stuschke et al., "Phase III comparison of preoperative chemotherapy compared with chemoradiotherapy in patients with locally advanced adenocarcinoma of the esophagogastric junction," Journal of clinical oncology, vol. 27, no. 6, pp. 851-856, 2009.

[35] S. Lorenzen, P. Thuss-Patience, S. E. Al-Batran et al., "Impact of pathologic complete response on disease-free survival in patients with esophagogastric adenocarcinoma receiving preoperative docetaxel-based chemotherapy," Annals of oncology, vol. 24, no. 8, pp. 2068-2073, 2013.

[36] M. L. Sánchez de Molina, C. Díaz Del Arco, P. Vorwald, D. García-Olmo, L. Estrada, and M. J. Fernández-Aceñero, "Histopathological factors predicting response to neoadjuvant therapy in gastric carcinoma," Clinical and Translational Oncology, vol. 20, no. 2, pp. 253-257, 2018.

[37] Y. H. Chen, J. Xiao, X. J. Chen et al., "Nomogram for predicting pathological complete response to neoadjuvant chemotherapy in patients with advanced gastric cancer," World journal of gastroenterology, vol. 26, no. 19, pp. 2427-2439, 2020.

[38] A. Guner and R. Yildirim, "Surgical management of metastatic gastric cancer: moving beyond the guidelines," Translational gastroenterology and hepatology, vol. 4, p. 58, 2019.

[39] K. Yoshida, K. Yamaguchi, N. Okumura, T. Tanahashi, and Y. Kodera, "Is conversion therapy possible in stage IV gastric cancer: the proposal of new biological categories of classification," Gastric cancer, vol. 19, no. 2, pp. 329-338, 2016.

[40] A. Biondi, R. Persiani, F. Cananzi et al., "R0 resection in the treatment of gastric cancer: room for improvement," World journal of gastroenterology, vol. 16, no. 27, pp. 3358-3370, 2010.

[41] Y. J. Bang, Y. W. Kim, H. K. Yang et al., "Adjuvant capecitabine and oxaliplatin for gastric cancer after D2 gastrectomy (CLASSIC): a phase 3 open-label, randomised controlled trial," Lancet, vol. 379, no. 9813, pp. 315-321, 2012.

[42] D. L. Ren, J. Li, H. C. Yu et al., "Nomograms for predicting pathological response to neoadjuvant treatments in patients with rectal cancer," World journal of gastroenterology, vol. 25, no. 1, pp. 118-137, 2019.

[43] E. L. Toxopeus, D. Nieboer, J. Shapiro et al., "Nomogram for predicting pathologically complete response after neoadjuvant chemoradiotherapy for oesophageal cancer," Radiotherapy and Oncology, vol. 115, no. 3, pp. 392-398, 2015. 